\title{
Cervical myelitis in a patient with pulmonary sarcoidosis
}

Eun Joo Chung, $\mathrm{MD}^{1,2}$; So-Young Lee, $\mathrm{MD}^{3}$; Jin-Hyung Lee, $\mathrm{MD}^{3}$; Yoon Ah Park, MD ${ }^{3}$; Bong Kwon Chun, $\mathrm{MD}^{4}$; So-Young Huh, $\mathrm{MD}^{3}$

'Department of Neurology, Inje University Busan Paik Hospital, Inje University College of Medicine, Busan, Republic of Korea

${ }^{2}$ Dementia and Neurodegenerative Disease Research Center, Inje University, Gimhae, Republic of Korea

${ }^{3}$ Department of Neurology, Kosin University College of Medicine, Busan, Republic of Korea

${ }^{4}$ Department of Pathology, Kosin University College of Medicine, Busan, Republic of Korea

\section{Journal of Neurocritical \\ CASE REPORT}

Received: November 14, 2019

Revised: December 11, 2019

Accepted: December 25, 2019

Corresponding Author:

So-Young Huh, MD

Department of Neurology, Kosin

University College of Medicine, 262

Gamcheon-ro, Seo-gu, Busan 49267,

Republic of Korea

Tel: +82-51-990-6461

Fax: +82-51-990-3077

E-mail: caccu@naver.com

Background: Sarcoidosis is a multisystemic disease characterized by noncaseating granulomas, predominantly affecting organs such as the lungs and lymph nodes. Spinal cord involvement of sarcoidosis is uncommon.

Case Report: A 32-year-old male presented with pain and numbness in bilateral upper extremities for 1 year. He had pulmonary sarcoidosis 4 years ago. Spinal magnetic resonance imaging showed a cord lesion with enhancement at the C5 to C6 level. Chest computed tomography revealed the increased size of the lymph nodes compared to previously. His serum angiotensin-converting enzyme level was elevated. He was diagnosed with myelitis caused by sarcoidosis. After steroid treatment, the numbness improved, but the pain still persisted.

Conclusion: To enable the early detection and treatment of neurosarcoidosis among patients with systemic sarcoidosis, a high degree of suspicion is required. Neurological complications can be minimized if it is detected and treated early.

Keywords: Neurosarcoidosis; Myelitis; Spinal cord

\section{INTRODUCTION}

Sarcoidosis is a multisystemic disease characterized by noncaseating granulomas, that predominantly affect organs such as the lungs and lymph nodes. The neurological manifestation of sarcoidosis has been described in $5 \%$ to $10 \%$ of patients $[1,2]$. Neurosarcoidosis can involve the cranial nerves, meninges, brain parenchyma, spinal cord, dura, muscle, and peripheral nerves. Spinal cord involvement, including intramedullary and extramedullary lesions, is uncommon [3]. Here, we report a patient with cervical myelitis and a previous diagnosis of pulmonary sarcoidosis. His clinical course, magnetic resonance imaging (MRI) findings, and clinical management are described.

\section{CASE REPORT}

A 32-year-old male presented with a 1-year history of pain in his neck, arms, and hands. He also complained bilateral numbness of his upper extremities 2 months prior to admission.

Four years ago, he had no pulmonary symptoms, but bilateral hilar and lower paratracheal lymphadenopathy was incidentally discovered during a routine medical chest radiography and com-

(C) 2020 The Korean Neurocritical Care Society

This is an Open Access article distributed under the terms of the Creative Commons Attribution Non-Commercial License (http://creativecommons.org/licenses/by-nc/4.0/) which permits unrestricted noncommercial use, distribution, and reproduction in any medium, provided the original work is properly cited. 

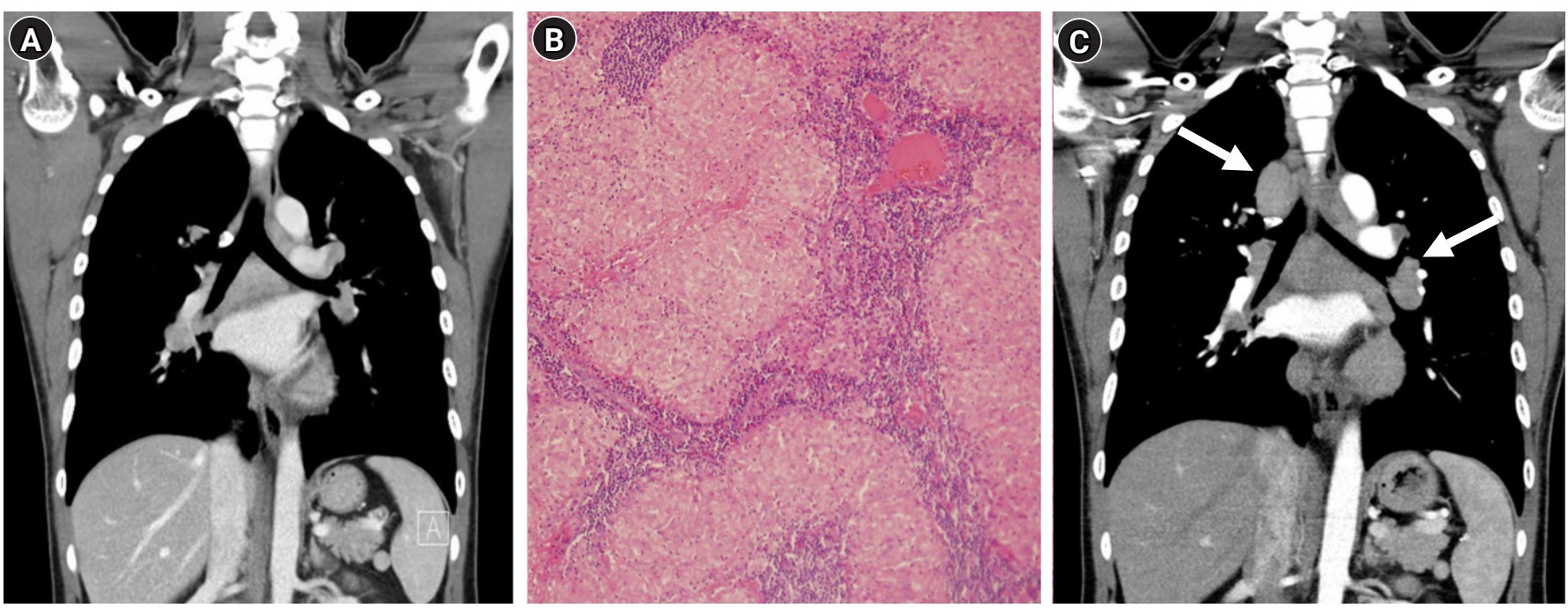

Fig. 1. (A) Four years ago, contrast-enhanced chest computed tomography (CT) showing enlargement of multiple lymph nodes. (B) Histopathology showing noncaseating granulomatous inflammation (hematoxylin and eosin stain, $\times 100$ ). (C) At the time of diagnosing cervical myelitis. Chest CT shows the slightly increased size of the paratracheal and hilar lymph nodes (arrows).

puted tomography (CT) (Fig. 1A). A biopsy via video-assisted thoracoscopic surgery showed noncaseating epithelioid granulomatous lesions compatible with pulmonary sarcoidosis (Fig. 1B). Tissue cultures for mycobacteria and fungus were negative. $\mathrm{He}$ was treated with low-dose oral steroids for 2 years but discontinued the medication arbitrarily.

On physical examination, the muscle strength in his limbs was normal. Sensory examination revealed that his light touch was decreased on the medial aspect of the bilateral upper arm, forearm, and hand. Deep tendon reflexes were normal. His visual analog scale (VAS) score was 7.

The findings of his nerve conduction studies, electromyography, median, and tibial nerve evoked potential studies we were normal. Brain MRI scan was normal. A spinal cord MRI showed T2 hyperintensity from C5 to C6, with focal patchy gadolinium enhancement between C5 and C6 (Fig. 2). Cerebrospinal fluid (CSF) was acellular with an elevated protein level $(52.2 \mathrm{mg} / \mathrm{dL})$. There were no oligoclonal bands in the CSF or serum. The immunoglobulin $\mathrm{G}(\mathrm{IgG})$ index was normal (0.52). CSF cytology was negative for malignancy. There were no oligoclonal bands in the CSF or serum. Test results for antinuclear antibody, antineutrophil cytoplasmic antibodies, aquaporin (AQP4) IgG, and myelin oligodendrocyte glycoprotein (MOG) IgG were negative. Thyroid function and the serum IgG4 levels were within the normal range. His serum angiotensin-converting enzyme (ACE) level was elevated (79 U/L [normal range, 12 to 68]). A chest CT revealed increased enlargement of the hilar lymph nodes compared to the previous study (Fig. 1C).
He was diagnosed with a spinal cord manifestation of sarcoidosis and treated with intravenous methylprednisolone $(1,000 \mathrm{mg} /$ day, 3 days) followed by oral prednisolone. Azathioprine was administered in addition to prednisolone. At the 2-month follow-up, diminishing enhancement could be seen on the MRI (Fig. 3). The numbness had improved, but mild pain (VAS score, 3 ) persisted even though he was on continuous oral prednisolone (20 $\mathrm{mg}$ ) and azathioprine ( $75 \mathrm{mg}$ ) treatment.

This study was approved by the Institutional Review Board of Kosin University Gospel Hospital (KUGH 2020-03-026). Since this was a retrospective case study, the requirement for informed consent was waived.

\section{DISCUSSION}

Neurological manifestation occurs in 5\% to $10 \%$ of patients with sarcoidosis $[1,2]$. Spinal cord sarcoidosis rarely occurs, and intraspinal sarcoidosis is rarely reported ( $0.1 \%$ to $50 \%$ of neurosarcoidosis) [3]. However, neurologic involvement can manifest 2 to 3 years after the development of systemic sarcoidosis [4,5]. Additionally, autopsy studies show that up to half of pathologic neurosarcoidosis cases are not diagnosed during an individual's lifetime [6]. Therefore, the emergence of neurological symptoms in patients with systemic sarcoidosis should raise suspicion of neural involvement associated with sarcoidosis.

Diagnosis of spinal cord sarcoidosis can be challenging, given that a biopsy of the spinal cord is necessary to establish a "definitive" diagnosis of neurosarcoidosis (Table 1) [7]. Spinal cord bi- 

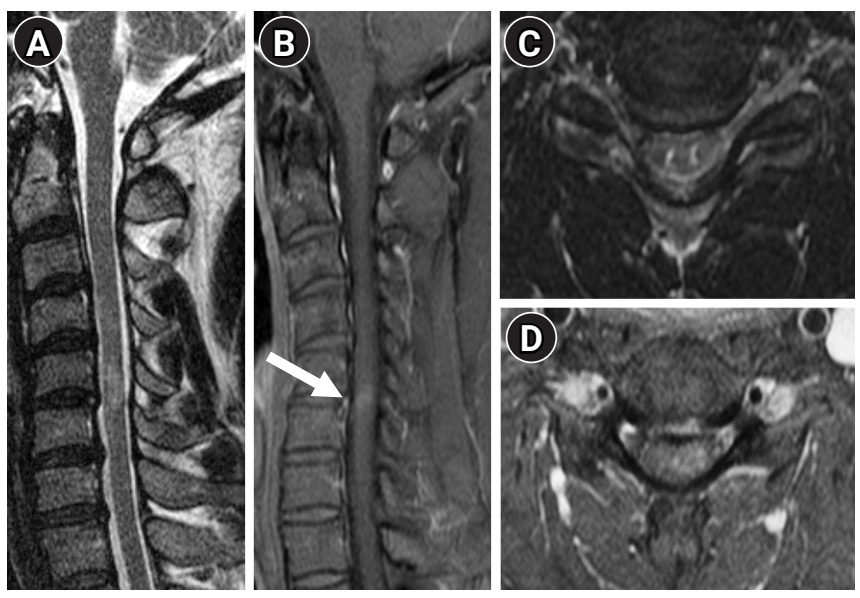

Fig. 2. (A) Sagittal T2-weighted magnetic resonance imaging (MRI) of the cervical spine showing a short segment of high signal intensity at the C5-C6 level. (B) T1-weighted gadolinium-enhanced image showing subtle enhancement between $\mathrm{C} 5$ and $\mathrm{C} 6$ segments (arrow). (C) Axial T2-weighted MRI showing bilaterally asymmetric linear foci of high T2-weighted signals. (D) Subtle enhancement can be seen on the left side of the spinal cord.
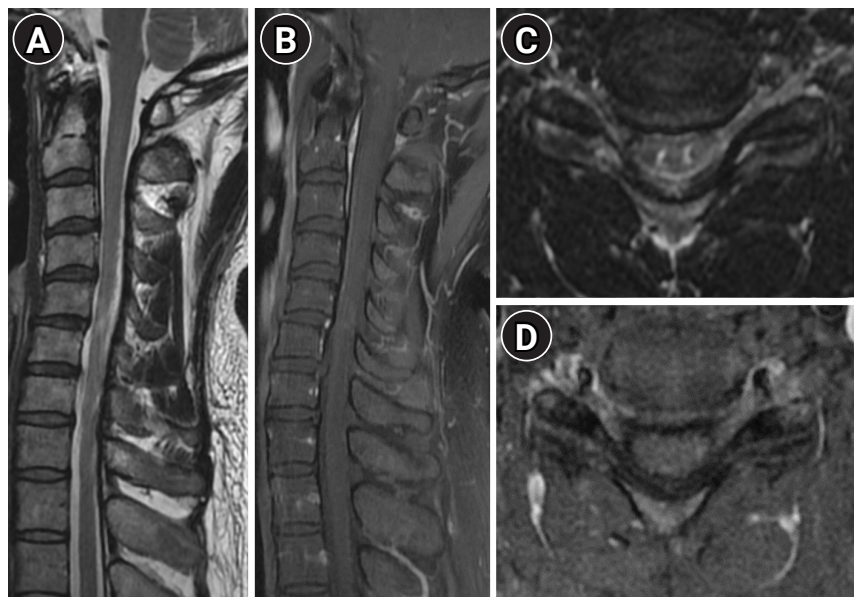

Fig. 3. Follow-up cervical spinal magnetic resonance imaging 2 months after intravenous methylprednisolone treatment. $(A, C)$ T2weighted images with a persistent high signal intensity. $(B, D)$ T1weighted gadolinium images showing diminished enhancement.

opsies can lead to increased morbidity and mortality [8]. Furthermore, biopsy samples do not always show abnormalities [8-10]. Our case had myelopathy with an elevated CSF protein level, elevated serum ACE level, positive histology of the hilar lymph node, and exclusion of alternative diagnoses, which led to the diagnosis of "probable" neurosarcoidosis [7]. Concerning differential diagnoses, the absence of brain lesions, oligoclonal bands, AQP4, and MOG antibodies and the enlargement of the hilar lymph node on the follow-up chest CT reduced the possibility of a demyelinating
Table 1. Proposed criteria for diagnosing neurosarcoidosis [7]

\begin{tabular}{cc}
\hline Definite & $\begin{array}{c}\text { Clinical presentation suggestive of neurosarcoidosis with } \\
\text { the exclusion of other possible diagnoses and the presence } \\
\text { of positive nervous system histology }\end{array}$ \\
Probable & $\begin{array}{c}\text { Clinical syndrome suggestive of neurosarcoidosis with } \\
\text { laboratory support for CNS inflammation (elevated levels } \\
\text { of CSF protein and/or cells, presence of oligoclonal bands, } \\
\text { and/or MRI evidence compatible with neurosarcoidosis) } \\
\text { and exclusion of alternative diagnoses together with } \\
\text { evidence for systemic sarcoidosis (through positive } \\
\text { histology, including Kveim test, and/or at least two } \\
\text { indirect indicators from a Gallium scan, chest imaging, } \\
\text { and serum ACE levels) } \\
\text { Clinical presentation suggestive of neurosarcoidosis with } \\
\text { the exclusion of alternative diagnoses where the above } \\
\text { criteria are not met }\end{array}$
\end{tabular}

Reprinted from Zajicek et al. [7], with permission of Oxford University Press. CNS, central nervous system; CSF, cerebrospinal fluid; MRI, magnetic resonance imaging; $A C E$, angiotensin-converting enzyme.

disorder of the central nervous system, such as multiple sclerosis and neuromyelitis optica.

In spinal cord sarcoidosis, clinical symptoms and MRI findings depend on the anatomic distribution and stage of the illness. There have been several MRI studies of spinal cord sarcoidosis involving lesions in the thoracic and cervical cord $[3,11]$. Most MRI findings can be relatively nonspecific, mimicking demyelinating disorders, as well as infectious and neoplastic diseases. An MRI analysis of 16 patients with intramedullary spinal sarcoidosis showed leptomeningeal enhancement, fusiform spinal cord enlargement, focal or diffuse intramedullary disease, and spinal cord atrophy depending on the clinical course [11]. Additionally, gadolinium enhancement occurred in the spinal cord even during the chronic phase of the disease course [11], similar to the heterogeneous intramedullary gadolinium enhancement seen in this case 1 year after symptom onset. This intramedullary T2 high signal intensity and faint enhancement without cord swelling may be related to a result of ischemia and disruption of the neural pathways by sarcoidosis in the chronic phase [11].

Our case showed elevated CSF protein without pleocytosis. Lymphocytic pleocytosis (40\% to $70 \%$ ), elevated protein ( $40 \%$ to $73 \%$ ), positive oligoclonal bands ( $22 \%$ to $55 \%$ ), and a low CSF glucose (10\% to $20 \%$ ) were reported in the CSF of neurosarcoidosis patients $[7,12,13]$. Wengert et al. [12] reported that CSF abnormalities in neurosarcoidosis are related with diffuse leptomeningeal enhancement on MRI and disease activity. Therefore, the CSF study may play a crucial role in assessing disease activity as well as a differential diagnosis.

In sarcoidosis, treatment is aimed at remission and symptom relief. Symptoms caused by inflammation are treated with corticosteroids, preferably in combination with immunosuppressants to 
prevent relapsing chronic sarcoidosis [14]. In this case, 2 years ago, the patient was lost to follow-up while on steroids. At the time of diagnosis of cervical myelitis, numbness recovered after the steroid pulse, but the patient continued to experience pain. At this point, systemic and periodic monitoring is essential to detect any subsequent inflammation to ensure timely treatment.

In conclusion, a high degree of suspicion of neurosarcoidosis is needed to enable early detection and treatment among patients with systemic sarcoidosis. Early detection and treatment will minimize neurological complications.

\section{ARTICLE INFORMATION}

\section{Conflict of interest}

No potential conflict of interest relevant to this article.

\section{ORCID}

Eun Joo Chung, https://orcid.org/0000-0001-8948-1135

So-Young Huh, https://orcid.org/0000-0002-3309-6155

Jin-Hyung Lee, https://orcid.org/0000-0003-2714-3266

Yoon Ah Park, https://orcid.org/0000-0003-3930-3319

Bong Kwon Chun, https://orcid.org/0000-0003-3565-9609

So-Young Lee, https: / / orcid.org/0000-0002-4576-3832

\section{Author contributions}

Conceptualization: EJC and SYH.

Data curation \& Formal analysis: SYL and BKC.

Visualization \& Writing-original draft: EJC, SYL, and SYH.

Writing-review editing: JHL and YAP.

\section{REFERENCES}

1. Stern BJ, Krumholz A, Johns C, Scott P, Nissim J. Sarcoidosis and its neurological manifestations. Arch Neurol 1985;42:90917.

2. Culver DA, Ribeiro Neto ML, Moss BP, Willis MA. Neurosarcoidosis. Semin Respir Crit Care Med 2017;38:499-513.

3. Cohen-Aubart F, Galanaud D, Grabli D, Haroche J, Amoura Z,
Chapelon-Abric C, et al. Spinal cord sarcoidosis: clinical and laboratory profile and outcome of 31 patients in a case-control study. Medicine (Baltimore) 2010;89:133-40.

4. Inoue $\mathrm{Y}$, Inui N, Hashimoto D, Enomoto N, Fujisawa T, Nakamura $\mathrm{Y}$, et al. Cumulative incidence and predictors of progression in corticosteroid-naïve patients with sarcoidosis. PLoS One 2015; 10:e0143371.

5. Christoforidis GA, Spickler EM, Recio MV, Mehta BM. MR of CNS sarcoidosis: correlation of imaging features to clinical symptoms and response to treatment. AJNR Am J Neuroradiol 1999;20:655-69.

6. Iwai K, Tachibana T, Takemura T, Matsui Y, Kitaichi M, Kawabata Y. Pathological studies on sarcoidosis autopsy. I. Epidemiological features of 320 cases in Japan. Acta Pathol Jpn 1993; 43:372-6.

7. Zajicek JP, Scolding NJ, Foster O, Rovaris M, Evanson J, Moseley IF, et al. Central nervous system sarcoidosis: diagnosis and management. QJM 1999;92:103-17.

8. Kumar N, Frohman EM. Spinal neurosarcoidosis mimicking an idiopathic inflammatory demyelinating syndrome. Arch Neurol 2004;61:586-9.

9. Kanoff RB, Ruberg RL. Intramedullary sarcoidosis of the spinal cord: report of a case. J Am Osteopath Assoc 1978;77:868-75.

10. Vighetto A, Fischer G, Collet P, Bady B, Trillet M. Intramedullary sarcoidosis of the cervical spinal cord. J Neurol Neurosurg Psychiatry 1985;48:477-9.

11. Junger SS, Stern BJ, Levine SR, Sipos E, Marti-Masso JF. Intramedullary spinal sarcoidosis: clinical and magnetic resonance imaging characteristics. Neurology 1993;43:333-7.

12. Wengert O, Rothenfusser-Korber E, Vollrath B, Bohner G, Scheibe F, Otto C, et al. Neurosarcoidosis: correlation of cerebrospinal fluid findings with diffuse leptomeningeal gadolinium enhancement on MRI and clinical disease activity. J Neurol Sci 2013;335:124-30.

13. Lacomis D. Neurosarcoidosis. Curr Neuropharmacol 2011;9: 429-36.

14. Grunewald J, Grutters JC, Arkema EV, Saketkoo LA, Moller DR, Müller-Quernheim J. Sarcoidosis. Nat Rev Dis Primers 2019;5:45. 